\title{
Chapter 10 \\ Multiorgan Segmentation Using Coherent Propagating Level Set Method Guided by Hierarchical Shape Priors and Local Phase Information
}

\author{
Chunliang Wang and Örjan Smedby
}

\begin{abstract}
In this chapter, we introduce an automatic multiorgan segmentation method using a hierarchical-shape-prior-guided level set method. The hierarchical shape priors are organized according to the anatomical hierarchy of the human body, so that the children structures are always contained by the parent structure. This hierarchical approach solves two challenges of multiorgan segmentation. First, it gradually refines the prediction of the organs' position by locating and segmenting the larger parent structure. Second, it solves the ambiguity of boundary between two attaching organs by looking at a large scale and imposing the additional shape constraint of the higher-level structures. To improve the segmentation accuracy, a model-guided local phase term is introduced and integrated with the conventional region-based energy function to guide the level set propagation. Finally, a novel coherent propagation method is implemented to speed up the model-based level set segmentation. In the VISCERAL Anatomy challenge, the proposed method delivered promising results on a number of abdominal organs.
\end{abstract}

C. Wang $(\varangle) \cdot$ Ö. Smedby

Center for Medical Image Science and Visualization (CMIV), Linköping University,

Linköping, Sweden

e-mail: chunliang.wang@liu.se

Ö. Smedby

e-mail: orjan.smedby@sth.kth.se

C. Wang · Ö. Smedby

Department of Radiology and Department of Medical and Health Sciences,

Linköping University, Linköping, Sweden

C. Wang · Ö. Smedby

School of Technology and Health (STH), KTH Royal Institute of Technology,

Stockholm, Sweden

(C) The Author(s) 2017

A. Hanbury et al. (eds.), Cloud-Based Benchmarking

of Medical Image Analysis, DOI 10.1007/978-3-319-49644-3_10 


\subsection{Introduction}

Shape-prior-guided image segmentation methods are popular choices for various challenging segmentation tasks $[4,5,7,13,18]$. This is because the constraint from shape priors substantially reduces the risk of region leaking that could occur often if the segmentation algorithm merely relies on image features. However, in our experience, this constraint is no guarantee for successful segmentation, as undesired segmentation errors can still occur due to non-ideal model initialization or weak organ edge discrimination, which often happens when two neighbouring organs have similar intensity. Figure $10.1 \mathrm{~b}$ shows an example of such failed shape-prior-guided segmentation. In this non-contrast CT scan, the liver model is misled towards the heart and chest wall by the similar intensity of these organs and the lack of gradient at the organ boundaries. This error could potentially be corrected if the segmentation algorithm incorporates some more sophisticated edge filters/detectors to enhance the vague borders between organs [9, 25]. However, such efforts often result in unstable solutions that will only work for a certain type of image and are sensitive to image quality and intensity variations. On the other hand, when asking a medical expert to perform the same task, such "absurd" errors will never happen, as the human observer has already identified the heart area and chest wall using his/her anatomical knowledge. In other words, the expert is doing a multiorgan segmentation even when he/she is asked to perform a single-organ segmentation. This led us to a simple philosophy: when it is difficult to tell whether a voxel belongs to organ A or not, it is probably easier to check whether it belongs to organ B or C. Based on this philosophy and the hierarchical nature of the human anatomy, we developed a hierarchical-shapeprior-guided multiorgan segmentation method. In the hierarchical shape model, the major structures with less population variation are at the top and smaller structures

(a)

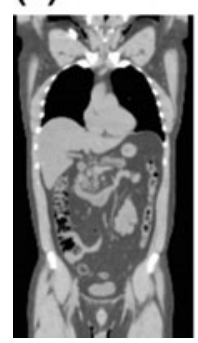

(b)

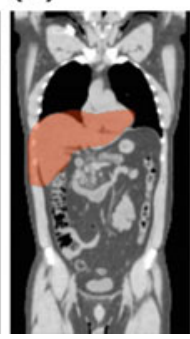

(c)

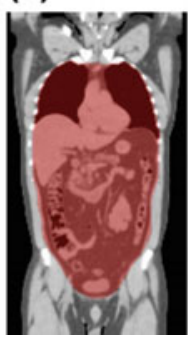

(d)

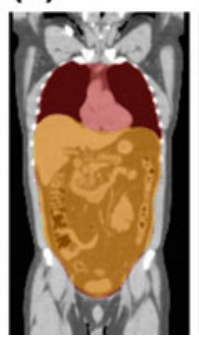

(e)

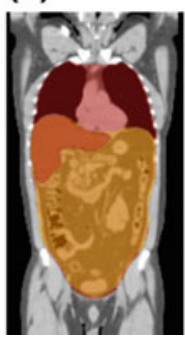

(f)

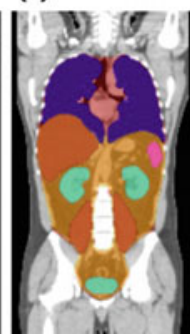

Fig. 10.1 A comparison between the conventional single-organ segmentation method (b) and the proposed hierarchical multiorgan approach $(\mathbf{c}-\mathbf{f})$. a Coronal view of a non-enhanced CT scan (note that the intensities of the liver, chest wall and heart are almost identical). b Failed liver segmentation with a single-organ shape prior. $\mathbf{c}$ Shape-prior-based ventral cavity segmentation. d Abdominopelvic cavity segmentation (yellow) on top of the ventral cavity (red). e Liver segmentation (brown) on top of ventral and abdominopelvic cavity segmentation. f Final multiorgan segmentation result. (Images are from our previous publication [17]) 
with higher irregularities are linked at a lower level. As shown in Fig. 10.1c-e, the segmentation starts from a large scale, so that the border between the ventral cavity and the chest and abdominal wall (Fig. 10.1c) is delineated, and then, at a smaller scale the, border between the thoracic and abdominal cavity (Fig. 10.1d) is identified. At the finest scale, individual organs are segmented (Fig. 10.1e).

The proposed top-down approach solves two major challenges of organ segmentation. The first one is to locate the anatomical structures within the dataset. Due to respiratory motion and anatomical variation, even for the scans with similar scanning range, the location of the same organ can still vary considerably. In the proposed multiorgan segmentation framework, the location information of the major structures is first detected with higher confidence and then passed down to the lower-level structures to initialize their segmentation. This process is similar to a multi-resolution registration approach. However, the benefit of using statistical shape models at each level is that the negative influence of anatomical and appearance variation of finer structures is eliminated to a large extent. The other major challenge that the proposed method solves is to delineate the boundary between two closely attached organs. Such delineation can be difficult in certain places where the contrast between organs is very vague or vanishes. In addition to the local features, the proposed method also utilizes the shape information of larger structures to guide the segmentation, i.e. the boundary information from higher-level structures provides extra cues to guide the segmentation of the lower-level structures. Such a hierarchical framework has proved to be very robust and performed relatively well even on non-contrast-enhanced CT image when using only region-based energy based on image intensity $[16,17]$.

To further improve the segmentation accuracy of the hierarchical model-based method, a model-guided edge-based energy term is proposed and combined with the region-based energy term to guide the level set evolution [19]. Unlike the conventional edge-based energy terms, which ignore the orientation of the edge-related features, the model-guided edge-based energy term uses the normal direction of the shape model to suggest the searching orientation of the local structures. This makes it possible to distinguish the black-to-white edges from white-to-black edges, which generate the same edge responses when using conventional gradient and local phase measurements. As such ambiguity often exists in the area where two organs' borders approach each other, there is a greater chance for the segmentation region to leak to the nearby organ when using conventional edge-based energy terms.

Finally, to improve segmentation speed, a novel coherent propagating level set algorithm was implemented. The new algorithm forces the contour to move monotonically according to a predicted developing trend which makes the level set functions converge faster. It also makes it possible to detect local convergence, so that the parts of the boundary that have reached their final position can be excluded in subsequent iterations, thus significantly reducing computation time [20, 22]. The proposed method was tested using the VISCERAL benchmark database, and promising results were delivered within reasonable processing time without any user intervention. 


\subsection{Statistical Shape-Prior-Guided Level Set Segmentation}

As a member of the active contour family, the level set method segments the targeted object in an image by propagating an initial contour towards the object's border. The movement of the contour is usually guided by two types of forces: the external and internal forces. While the external force is often designed to drive the contour towards the object's border, the internal force is commonly designed to keep the contour smooth. To generate these forces, the segmentation problem is often formulated as an energy minimization problem, as demonstrated in Eq. 10.1, where $\phi$ is the level set function, and $\alpha, \beta$ are weighting factors.

$$
E(\phi)=\alpha E_{\text {in }}(\phi)+\beta E_{\text {ex }}(\phi)
$$

The most common external energy functions include region-based image energy and gradient-based image energy; examples are given in Eqs. 10.2 and 10.3, respectively. The former converts the input image intensity into probability functions of a pixel/voxel belonging to the object or the background [3], while the latter utilizes the image gradient to guide the movement of the contour so that the contour is attracted to areas with higher gradient [2].

$$
\begin{gathered}
E_{\text {region }}(\phi)=-\int \log \left[p_{A}(I(x))\right] H(\phi(x)) d x-\int \log \left[p_{B}(I(x))\right](1-H(\phi(x))) d x \\
E_{\text {edge }}(\phi)=\int(|\nabla I(x)|+1)^{-1} \phi^{\prime}(x) d x \\
E_{\text {in }}(\phi)=\int|\nabla H(\phi(x))| d x
\end{gathered}
$$

Here, $H$ is the Heaviside function, and $p_{A}$ and $p_{B}$ are probability functions of a pixel belonging to the object region and the background region, respectively. The internal energy is often connected with local curvature of the contour, which means it is minimized when the contour becomes smooth (e.g. Eq. 10.4). The local smoothness character of active contours makes them resistant to noise. The internal force can also, to some extent, prevent the segmentation region leaking to a neighbouring object through small "holes" that connect two regions with similar appearance. However, as the curvature is a local measurement, if the connecting "holes" are larger than the scale at which the smoothness is measured, leaking problems may still occur. To obtain the right segmentation results in such cases, we need to impose a stronger constraint on the shape of the contour. While there is no general solution to avoid the leaking problem for all cases, in medical images, we can often use the prior knowledge of the anatomical shape of the targeted structures. The statistical shape model-guided level set method proposed by Leventon et al. is an example of incorporating such shape prior knowledge into the image segmentation [13]. In this framework, a shape-based 
energy term is added to penalize the differences between the evolving contour and the shape prior (Eq. 10.5):

$$
E_{\text {mode }}(\phi)=\int(\phi(x)-m(t(x)))^{2} d x
$$

where $m$ represents the statistical shape model, and $t$ is a rigid transformation function.

How to represent the shape prior knowledge using mathematical models is still an active research field; a relatively complete review can be found in [5, 7]. In general, there are two types of statistical shape models that are commonly used for medical image segmentation. One is the mesh-based representations, such as the active shape models (ASMs) [4], where shapes are expressed as 2D contours or 3D meshes, and the variation of shapes is constrained by the distribution of the vertexes. Another type of shape models is region-based where the shapes are embedded in distance maps created from binary patterns. The latter is often used in the level setbased framework, since the level set function itself is also a distance map. For the mesh-based representations, it is less straightforward to define a transform function $t$ relating the meshes with the level set function. To create the region-based statistical model, manually created segmentation masks are first aligned using rigid registration; i.e., only translation, rotation and isotropic scaling are allowed. Then, these aligned binary masks are converted into signed distance maps via a distance transform. The prominent variations of these distance maps can be obtained via principal component analysis (PCA). Finally, the statistical model is represented by combining the mean of the signed distance maps $(\bar{m})$ and a weighted combination of the variation maps $\left(m_{\sigma 1}, m_{\sigma 2}, \ldots m_{\sigma n}\right)$ (Eq. 10.6).

$$
M=\bar{m}+\omega_{1} m_{\sigma 1}+\omega_{2} m_{\sigma 2}+\cdots+\omega_{n} m_{\sigma n}
$$

It should be pointed out that the PCA process mentioned above does not guarantee the algorithm to fully recover the population variation of the targeted shape. This is because the distance transform is a nonlinear process. It has been shown that in some cases, the shape variation is highly nonlinear [5]. This limits these types of methods to shapes that do not vary in a too complicated manner. Fortunately, this is often not a big problem for anatomical structures in medical applications.

Combining the region-based term and the model-based term as the external energy leads us to the level set equation minimizing Eq. 10.1:

$$
\frac{\partial \phi}{\partial t}=\left[\alpha \operatorname{div}\left(\frac{\nabla \phi}{|\nabla \phi|}\right)+\beta\left(\log \left(p_{B}\right)-\log \left(p_{A}\right)\right)+\gamma m(t)\right]|\nabla \phi|
$$

The optimization of the level set function and the model fitting is usually performed iteratively in parallel; i.e., the model is re-estimated after one or several iterations of the level set evolution. The transformation $t$ and the weighting factors 
$\omega_{i}$ are usually solved by minimizing the squared distance between the model and the level set function, which is also a signed distance map.

\title{
10.3 Multiorgan Segmentation Using Hierarchical Shape Priors
}

\begin{abstract}
Although using shape priors prevents the leaking problem in many medical application, in some challenging cases, a single-organ model is still insufficient to generate a satisfactory segmentation result. Such failure can often be seen in cases where the connecting area between two organs of similar intensity is relatively large. An example is given in Fig. 10.1b. In this non-enhanced CT scan, the liver model was confused by the similar intensity between the liver and other surrounding organs. Even though there are visible gaps between them, such small local minima cannot prevent the model-based segmentation method to reach a global minimum when minimizing Eq. 10.1. While the connecting areas are relatively large for the liver, they become smaller when looking at a higher scale. For example, when looking at the ventral cavity (union of thoracic cavity and abdominopelvic cavity), there is no longer ambiguity of telling which is the chest wall and which is the liver (Fig. 10.1c). It is the same for the border between the heart and the liver when looking at the separation of the thoracic and abdominal cavity (Fig. 10.1d). Therefore, it is advantageous to use hierarchical shape priors that are organized according to the scale and shape complexity of different anatomical structures; i.e., the large organs of more regular shape with less interobject variation are located at the higher level, and the smaller structures of more complex appearance and variation are introduced at a lower level. During the segmentation, higher-level structures' location information will influence the lower-level structures' location, and the higher-level structures' border will also limit the area of the lower-level structures as the latter are assumed to be contained by their upper-level structures.
\end{abstract}

\subsubsection{Building Hierarchical Shape Priors}

The major structures of the proposed hierarchical shape priors are listed in Fig. 10.2. Building hierarchical shape priors consists of two major steps: building individual shape models and linking them together in a common space. The individual statistical shape model generation is not much different from the traditional statistical shape generation process described in Sect. 10.2. However, to make sure that the relative scale between different structures is preserved, a "standard patient" is selected in the beginning via a visual comparison. Organs that are manually segmented from other patients are all registered towards the corresponding organ of this standard patient. 


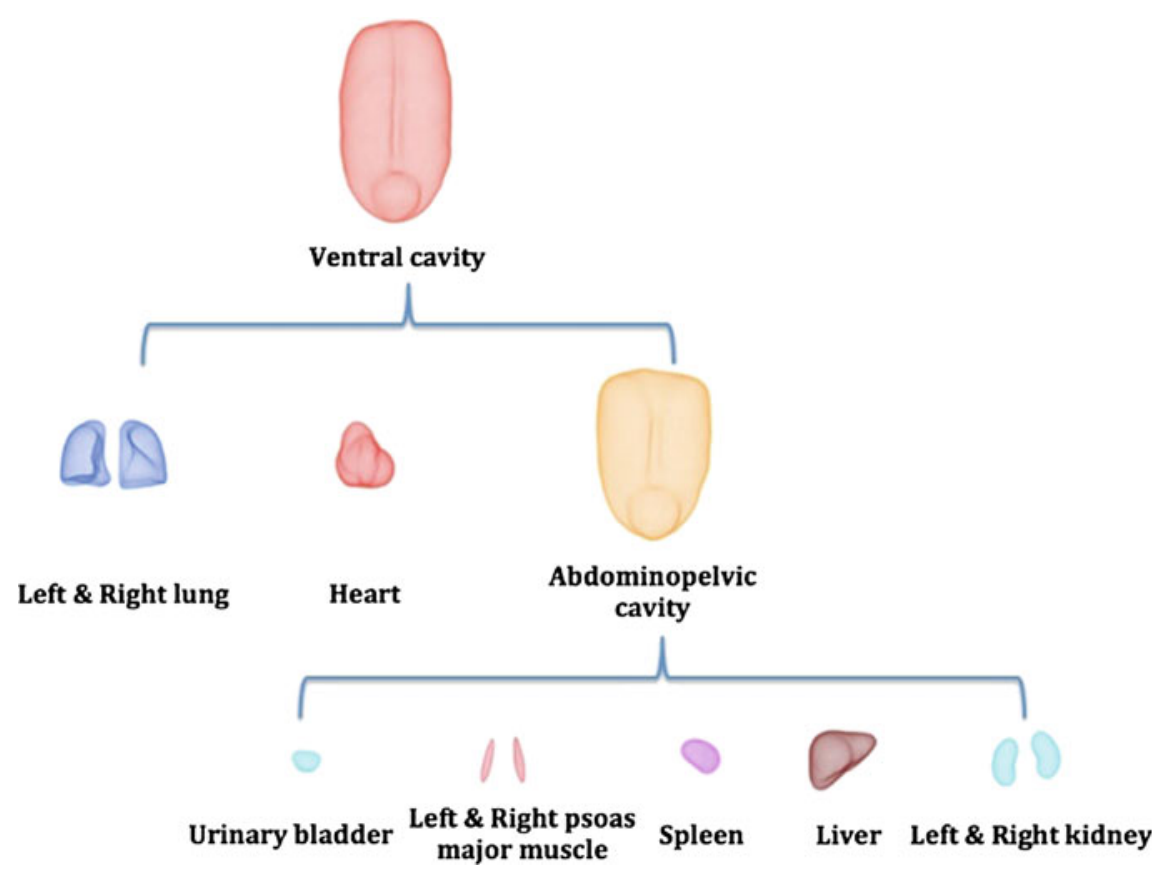

Fig. 10.2 An overview of the hierarchical organization of the shape priors

To link different organs and structures to a common space, the children-level structures are projected into their parent-level structure's space using the transformation matrices that align the upper-level structures. For example, after the ventral cavity masks of all other subjects are registered towards the picked subject's ventral cavity, the children-level structures of those subjects, i.e. lungs, heart and abdominopelvic cavity, are projected to the mean shape of the ventral cavity using the same rigid transform as the ventral cavities. These structures will not be perfectly aligned, but the sum of the masks will form a probability map that suggests the likelihood of the corresponding organ appearing at a certain place of the ventral cavity. A binary trust zone of the lower-level organ is created by setting a threshold on the probability map. Finally, the mean shapes of these children structures are registered towards the corresponding trust zones. Through these chains of transformation, we establish a rigid transform from the mean shape of a children structure to its parent structure. These transformation matrices are used to initialize the position of the children structure models, once the segmentation of their parent structures is finished. Figure 10.3 shows the mean shapes of all structures used in our hierarchical shape model and their relative positions determined using the approach describe here. 

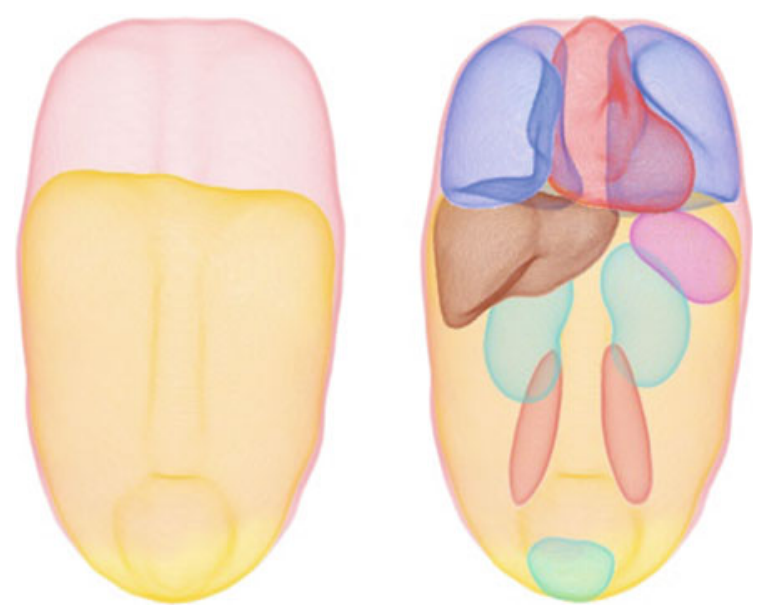

Fig. 10.3 The mean shape of different anatomical structures and their relative positions

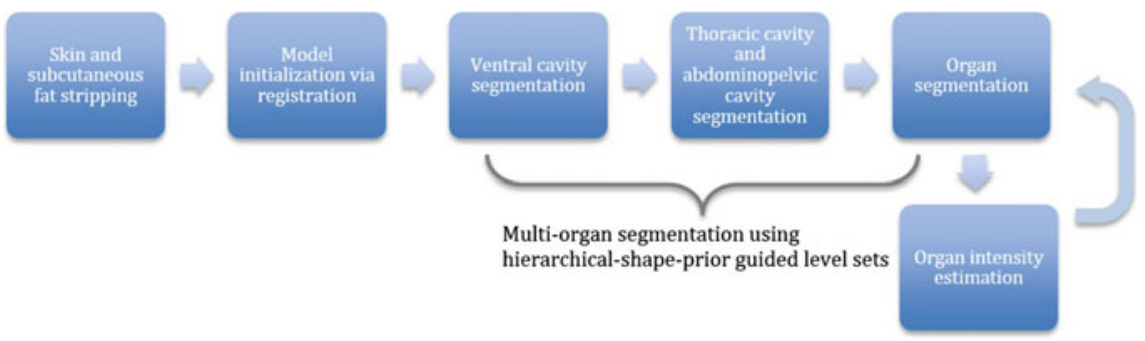

Fig. 10.4 The processing pipeline of the proposed multiorgan segmentation framework

\subsubsection{Multiorgan Segmentation Using Hierarchical Shape Priors}

Figure 10.4 summarizes the processing pipeline of the proposed segmentation framework. In a preprocessing step, we try to remove the skin and subcutaneous fat from the patient's image data using a threshold-based level set method combined with mathematical morphological operations. Because these tissues vary considerably from patient to patient, if present, they may mislead the following registration between the unseen patient and the standard patient mentioned above. This registration step is designed to initialize the ventral cavity model's position in the unseen patient. The segmentation steps are performed in a top-down fashion guided by the hierarchical shape model; i.e., the ventral cavity is first segmented and then divided into thoracic cavity and abdominopelvic cavity. At a third stage, the individual organs such as liver, spleen and kidneys are segmented. Within the same level, structures are segmented sequentially from left to right in the order listed in Fig. 10.2. 
The segmentation process for individual structures is very similar to the modelguided level set method described in Sect. 10.2, except that an additional energy term is added to let the parent structure regulate the children structures' segmentation. The new energy function is given in Eq. 10.8.

$$
E_{\text {parent_mode }}(\phi)=\int\left(1-H\left(\phi_{p}(x)\right)-H(\phi(x))\right)^{2} d x
$$

Here, $\phi_{p}$ is the segmentation result of the parent structure. For the ventral cavity, $\phi_{p}$ is set to be the level set function of the subcutaneous fat stripping step. Note that as long as the children structure is inside its parent, the latter has no influence on the lower-level structure segmentation. Besides this regulation force, the segmentation of the parent structure also provides the initial position of the children structures using the relative transformation matrix obtained in the hierarchical shape model training phase.

\subsubsection{Region-Based External Speed Function}

Besides the model terms, the image term of the level set function is another important factor of the multiorgan framework. As presented in Sect. 10.2, common external speed functions include region-based methods and gradient-based methods. In our preliminary implementation of the proposed hierarchical-shape-prior-guided level set framework, we chose to use the region-based approach, where the external speed function is an intensity mapping function. Like the threshold-based level set method proposed by Lefohn et al. [12], the mapping function outputs a positive speed if the CT value is close to the mean intensity of a selected organ, but a negative speed when the CT value is unlikely to be seen in that organ. For non-contrast-enhanced CT scans, the intensity distribution of most parenchymal organs is relatively consistent from patient to patient, which allows us to use the same sets of parameters to guide the segmentation of the same organ in different subjects. Some examples are plotted in Fig. 10.5. Note that as the intensity distribution of some neighbouring organs/structures may overlap with the targeted organ, the ceiling and floor of the mapping function are manually tuned to avoid leaking into the neighbouring structures. This results in an asymmetric intensity mapping function, like for the ventral cavity. For contrast-enhanced CT scans, the intensity distribution of some organs may vary significantly depending on the circulation rate and acquisition timing, and an iterative intensity range estimation approach is then used for segmenting the heart, liver, kidney and spleen. Using the imperfect initial segmentation, we estimate the mean intensity $(M)$ and its standard deviation $(\sigma)$ of the targeted organ. The organ's upper and lower thresholds are then set to be $M+1.5 \sigma$ and $M-1.5 \sigma$, respectively. To avoid the influence of undesired tissue included in these preliminary segmentations, all voxels with intensity lower than $30 \mathrm{HU}$ are excluded from the calculation 
Fig. 10.5 Example thresholding functions for the image term

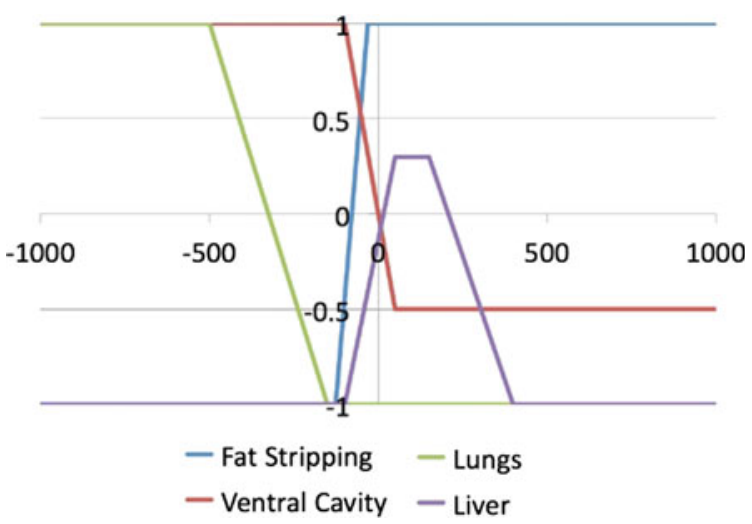

of $M$ and $\sigma$. The intensity estimation is repeated during the model fitting process. The iterative intensity estimation stops when the changing rates of $M$ and $\sigma$ are both lower than a threshold (5 HU).

\title{
10.4 Improving Segmentation Accuracy Using Model-Guided Local Phase Analysis
}

\begin{abstract}
Although the region-based speed function alone can generate relatively good segmentation results, in complicated cases where the organ is attached to several neighbouring structures with various intensity ranges, it is advantageous to rely not only on the image intensity but also on the edge information. Therefore, combining the region-based and gradient-based speed function is a natural way to improve the segmentation accuracy. However, conventional gradient-based edge delineation cannot distinguish between black-to-white and white-to-black edges. In medical images, we often see two organs approaching each other in some areas. As both edges generate high gradient measurements, the contour may be attracted to either side depending on the initial position of the contour. To avoid such ambiguity, we have proposed a model-guided local phase analysis that is able to distinguish between these two types of edges and avoid the segmentation region leaking into another organ.
\end{abstract}

\subsubsection{Quadrature Filters and Model-Guided Local Phase Analysis}

So-called quadrature filters are pairs of filters designed to measure whether the underlying image structure is similar to a ridge-like pattern or an edge-like pattern. An example of a two-dimensional quadrature pair set is shown in Fig. 10.6a, b. The 

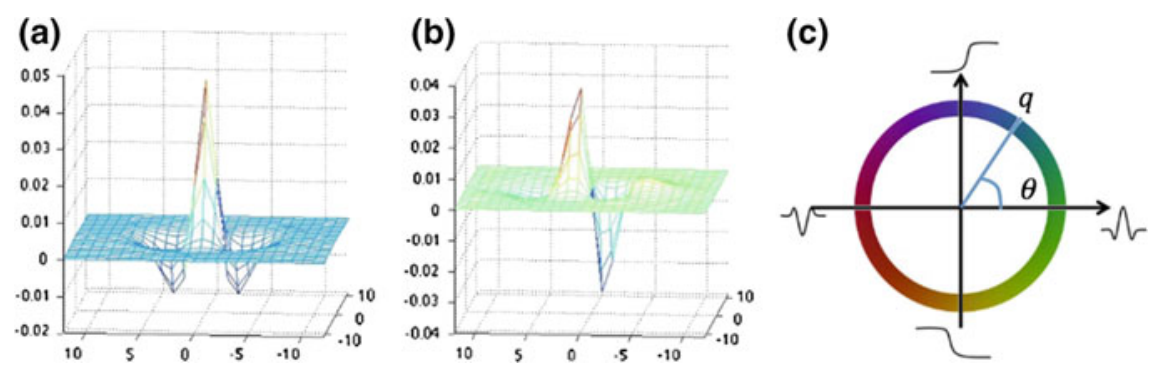

Fig. 10.6 An example of quadrature filter pairs in 2D. a The ridge-picking filter. $\mathbf{b}$ The edge-picking filter. $\mathbf{c}$ The quadrature filter's response in the complex plane

output of this pair of filters is represented by a complex number where the real part is the output of the ridge-picking filter and the imaginary part is the output of the edge-picking filter. The argument of this complex number in the complex plane is referred to as the local phase [10], $\theta$ in Fig. 10.6c. The magnitude $(q)$ of the complex number is called local energy [10]. Local phase is often used as a promising alternative to image gradient for image segmentation $[1,11,18]$. This is because the phase measure measurement changes monotonically when moving from one side of the edge to the other side, which makes it easier to design a speed function. However, like gradient measurements, local phase is also orientation-dependent. In practice, the local phase is often estimated using the local orientation estimated from the local gradient or eigenvectors of the local structure tensor. These types of solutions will produce the same phase for black-to-white and white-to-black edges. When used for segmentation, the local phase still cannot prevent the contour from leaking to the edge of a neighbouring organ.

Here, we propose to use the evolving statistical shape model, instead of the input image, to generate the reference direction for local phase measurement. This is done by converting the shape model into a signed distance map. When measuring the local phase at a point, the principle orientation of the quadrature filters is then aligned with the gradient of the signed distance map. Note that the reference orientation is perpendicular to the shape surface. More importantly, the gradient also indicates which direction is inside and outside. Unlike conventional local phase analysis, the output of the model-based phase analysis is $\pi / 2$ on a black-to-white edge and $-\pi / 2$ on a white-to-black edge. An example of applying the proposed phase analysis in a brain MRI volume is shown in Fig. 10.7h. Compared with the phase map produced by Läthén's method [11] (Fig. 10.7d), the proposed solution (Fig. 10.2h) makes a clear distinction between the inner boundary of the skull (blue) and the outer surface of the brain (yellow). 


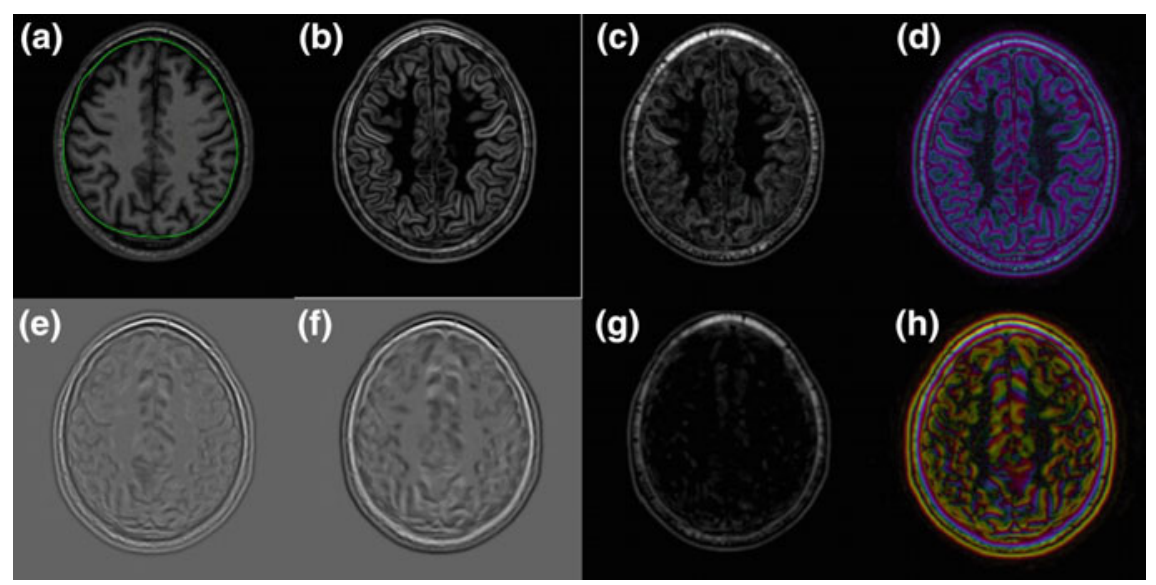

Fig. 10.7 a Input image: the green contour represents the cross section of the brain model; $\mathbf{b}$ the gradient magnitude; $\mathbf{c}, \mathbf{d}$ local energy and phase maps using Läthén's method [11]; $\mathbf{e}, \mathbf{f}, \mathbf{g}, \mathbf{h}$ the real and imaginary parts of the model-guided quadrature filter and corresponding local energy and phase maps. (d, $\mathbf{h}$ were created using the colour lookup table shown in Fig. 10.6c)

\subsubsection{Integrating Region-Based and Edge-Based Energy in the Level Set Method}

To use the phase information to guide the level set propagation, we propose an energy function as described in Eq. 10.9.

$$
E(\partial \mathscr{R})=\int_{0}^{1} g\left[\theta\left(I\left(\partial \mathscr{R}_{A}(c)\right)\right)-\tau\right]^{2}\left|\mathscr{R}_{A}(c)\right| d c
$$

Here, $I$ is the input image, $\mathscr{R}_{A}$ is the segmented region, and $\theta$ is the estimated local phase $(0 \leq \theta<2 \pi)$ at any given location. $\tau$ is the targeted phase (e.g. $\pi / 2$ for a black-to-white edge and $3 \pi / 2$ for a white-to-black edge). The function $g$ is simply a period-fixing function that ensures that the phase difference $\theta-\tau$ falls in the range from $-\pi$ to $\pi$ (Eq. 10.10).

$$
g(\delta)=\left\{\begin{array}{cc}
\delta & \text { if }-\pi<\delta \leq \pi \\
\delta-2 \pi & \text { if } \delta>\pi \\
\delta+2 \pi & \text { if } \delta \leq-\pi
\end{array}\right.
$$

Note that Eq. 10.9 is very similar to the conventional geodesic active contours given in Eq. 10.3, except that $\theta(I)$ replaces $\nabla I$, and the period-fixing function $g$ replaces the gradient magnitude inverse function. To combine the phase-based energy and the region-based energy, we propose an integrated energy function: 

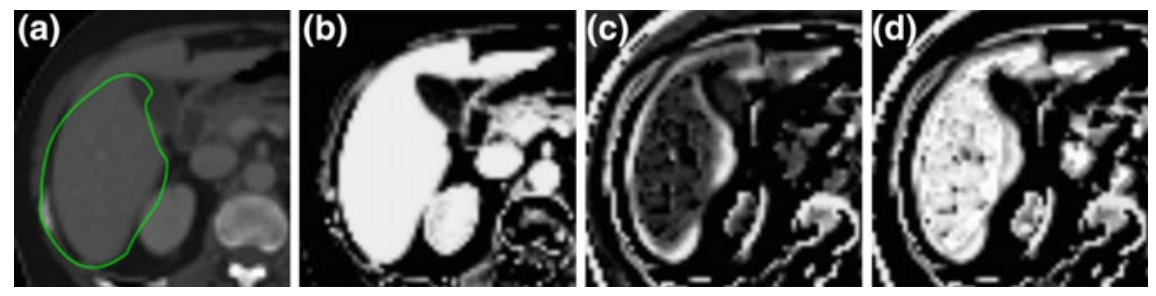

Fig. 10.8 a An axial view of an input volume (green contour shows the current shape model), (b) the region-based speed terms based on the image intensity, $\mathbf{c}$ the phase-based term estimated using the model, $\mathbf{d}$ the combined external speed map. Note that the connection between liver and kidney is removed

$$
\begin{aligned}
& E(\partial \mathscr{R})=\alpha \int_{0}^{1} g\left[\theta\left(I\left(\partial \mathscr{R}_{A}(c)\right)\right)-\tau\right]^{2}\left|\mathscr{R}_{A}(c)\right| d c- \\
& \int_{\mathscr{R}_{A}} \int w(x, y) \log \left[p_{A}(I(x, y))\right] \mathrm{dxdy}-\int_{\mathscr{R}_{B}} \int w(x, y) \log \left[p_{B}(I(x, y))\right] d x d y
\end{aligned}
$$

Here, $p_{A}$ and $p_{B}$ are the probability functions of a given pixel/voxel belonging to the region $A / B$. Function $w$ is a weighting function that weights the fitting energy using the local energy output $(q)$ from the quadrature filter, as described in Eq. 10.12:

$$
w(x, y)=\frac{1}{1+q(I(x, y))}
$$

The energy function is minimized by solving the following descent equation:

$$
\frac{\partial \phi}{\partial t}=\left[\alpha g(\theta-\tau)^{2} \operatorname{div}\left(\frac{\nabla \phi}{|\nabla \phi|}\right)+\alpha g(\theta-\tau)+w \log \left(p_{B}\right)-w \log \left(p_{A}\right)+m(t)\right]|\nabla \phi|
$$

Figure 10.8 shows an example of integrated speed in a liver segmentation case. The region-based and phase-based components of the speed function are also shown side by side. In practice, the first component on the right side can be replaced by $\alpha^{\prime} \operatorname{div}\left(\frac{\nabla \phi}{|\nabla \phi|}\right)$, where $\alpha^{\prime}$ is a weighting factor, as it is just a regulation term that corresponds to the curvature force in the conventional level set methods.

Since the filter orientation varies across the image, the filtering is made via local resampling by rotating a given kernel grid to align with the local reference direction. This step can also be carried out using a steerable filter, which synthesizes a filter with arbitrary orientation from a linear combination of basis filters [6]. The latter may be faster if the local phase analysis needs to be performed for all pixels/voxels. However, in practice, the computation on points that are far away from the model surface can be skipped, as will be further explained in the next section. 


\subsection{Speeding up Level Set Segmentation Using Coherent Propagation}

Level set methods are computationally intensive, and many efforts have been made to speed up level set-based segmentation. One common approach is to limit the computation to a narrow band around the zero level set. The most popular implementation of this type is the sparse field level set, which only updates the level set function on a 1-pixel wide band that the zero level set passes through [24]. However, the processing time is still in the range of $10-30$ min to segment a single organ from a voxel image [22]. To speed up level set segmentation, we proposed a fast level set method using coherent propagation [20, 22]. The new method not only limits the computation to a narrow band, but also eliminates the points that have reached the object border. This local convergence detection is enabled by synchronizing local points on the contour to move outwards or inwards together monotonically in a period instead of letting different points on a contour move outwards or inwards simultaneously. The speed function of the coherent propagating level set can be written as in Eq. 10.14 .

$$
\frac{\partial \phi}{\partial t}=W\left(V_{t}\right) \text { where } W\left(V_{t}\right)= \begin{cases}V_{t}(x) & \text { if } V_{t}(x) \operatorname{Tr}(x)>0 \\ 0 & \text { if } V_{t}(x) \operatorname{Tr}(x) \leq 0\end{cases}
$$

Here, $V_{t}$ is the conventional level set speed function (e.g. Eq. 10.7), and the function $\operatorname{Tr}$ represents a trend direction suggesting whether the local contour is expanding or shrinking. Within a single period, its value is fixed to 1 or -1 . The initial value of $\operatorname{Tr}$ can be either estimated using the average external speed in a neighbourhood [22] or assigned via user interaction, such as dragging the contour inwards or outwards [23]. In the latter case, the trend of the whole contour is synchronized, meaning that the contour is designed to only expand or shrink in the first period. A period ends when no points on the contour can move towards the trend direction. When entering the next period, the $T r$ function will switch sign at all points, and all points will propagate in the opposite direction until they stop again. The final segmentation result can be obtained after repeating the coherent propagation for a small number of periods (4-6). Within a single period, once $W(x)$ returns 0 , at point $x$, then this point is excluded from the further computation, until at least one of its neighbours' level set value has changed. The ability to detect the converged points helps the new level set method to achieve at least 10 times speedup in various segmentation tasks when compared with the sparse field level set algorithm [22].

Moving from level set segmentation to statistical shape model-guided level set segmentation, the computational burden becomes even greater. While simply plugging Eq. 10.7 as $v_{t}$ into Eq. 10.14 can already speed up the segmentation process considerably, another time-consuming part of model-based level set segmentation is to update the statistical model iteratively as the level set propagates. Although the narrowband strategy can also be applied here, i.e. using the voxel on the zero level set to drive the statistical shape fitting, the computation can still be slow if the fitting 
has to be repeated frequently. To reduce the frequency of updating the shape model, we limit the maximum travel distance of the active contour from the previous shape model by normalizing the image terms in Eq. 10.3 and tuning the weighting factors. This maximum travel distance is often set to be relatively small $(3-5 \mathrm{~mm})$, which means that the model fitting need not be repeated before the level set converges. When using the conventional level set method, it is not possible to know when the level set function will converge, and the level set evolution is often repeated a redundant number of iterations, which may outweigh the benefit of reducing the number of registrations. On the other hand, the coherent propagation method is capable of detecting the convergence by itself, thereby avoiding such redundant computations. In our preliminary experience of the new framework, the model fitting rarely needs to be repeated more than 20 times, given a relatively good initialization. The reduced model updating frequency will also directly benefit the model-guided phase computation, which can be very time-consuming, too. Moreover, since the contour is not allowed to move further than the maximum travel distance, we only need to compute the local phase for the points on the narrow band.

\subsection{Experiments and Results}

The proposed method was tested for multiorgan segmentation using the data from the VISCERAL multiorgan segmentation Benchmark. ${ }^{1}$ Our method was trained using 7 non-enhanced CT (CT) and 7 contrast-enhanced CT (CECT) datasets (the 14 training datasets from the VISCERAL Anatomy 1 challenge [8]) and tested on 8 non-enhanced and 10 enhanced CT datasets. In our experiments, we tested three implementations of the proposed method:

Implementation 1: Hierarchical model-guided multiorgan segmentation uses only the region-based speed function described in Sect. 10.3.3. The segmentation was made at a single resolution of $3 \mathrm{~mm}$ isotropic voxel size.

Implementation 2: Hierarchical model-guided multiorgan segmentation uses the combined local phase- and intensity-based speed function described in Sect. 10.4.2. The segmentation was done at a single resolution of $3 \mathrm{~mm}$ isotropic voxel size.

Implementation 3: Hierarchical model-guided multiorgan segmentation uses the combined local phase- and intensity-based speed function described in Sect. 10.4.2. The segmentation was done using a multi-resolution strategy, and the finest resolution was same as the input image.

Detailed results and comparison of these three implementations are listed in Table 10.1. We further tested the influence of two key parameters in the preprocessing step on the segmentation accuracy: the Gaussian smoothing kernel size and the downsample spacing. The results are plotted in Fig. 10.9. All these experiments were done using Implementation 1.

\footnotetext{
${ }^{1}$ VISCERAL Benchmark, http://www.visceral.eu/closed-benchmarks/anatomy2/anatomy2results/.
} 
Table 10.1 Multiorgan segmentation in CT and CECT datasets (mean Dice coefficient)

\begin{tabular}{l|l|l|l|l|l|l|l}
\hline Data & Method & Liver & $\begin{array}{l}\text { Right } \\
\text { Kidney }\end{array}$ & $\begin{array}{l}\text { Left } \\
\text { Kidney }\end{array}$ & Spleen & $\begin{array}{l}\text { Right } \\
\text { Lung }\end{array}$ & $\begin{array}{l}\text { Left } \\
\text { Lung }\end{array}$ \\
\hline CT (8 cases $)$ & Implementation 1 & $93.3 \%$ & $77.9 \%$ & $87.6 \%$ & $90.1 \%$ & $96.0 \%$ & $95.9 \%$ \\
& Implementation 2 & $93.2 \%$ & $71.7 \%$ & $88.8 \%$ & $91.0 \%$ & $96.3 \%$ & $95.9 \%$ \\
& Implementation 3 & $93.6 \%$ & $79.6 \%$ & $89.6 \%$ & $91.0 \%$ & $97.0 \%$ & $96.1 \%$ \\
\hline CECT (10 cases) $)$ & Implementation 1 & $92.9 \%$ & $92.2 \%$ & $92.6 \%$ & $87.0 \%$ & $96.6 \%$ & $96.6 \%$ \\
& Implementation 2 & $93.9 \%$ & $92.2 \%$ & $92.4 \%$ & $88.9 \%$ & $96.7 \%$ & $96.7 \%$ \\
& Implementation 3 & $94.9 \%$ & $95.9 \%$ & $94.5 \%$ & $90.9 \%$ & $97.1 \%$ & $97.2 \%$ \\
\hline
\end{tabular}
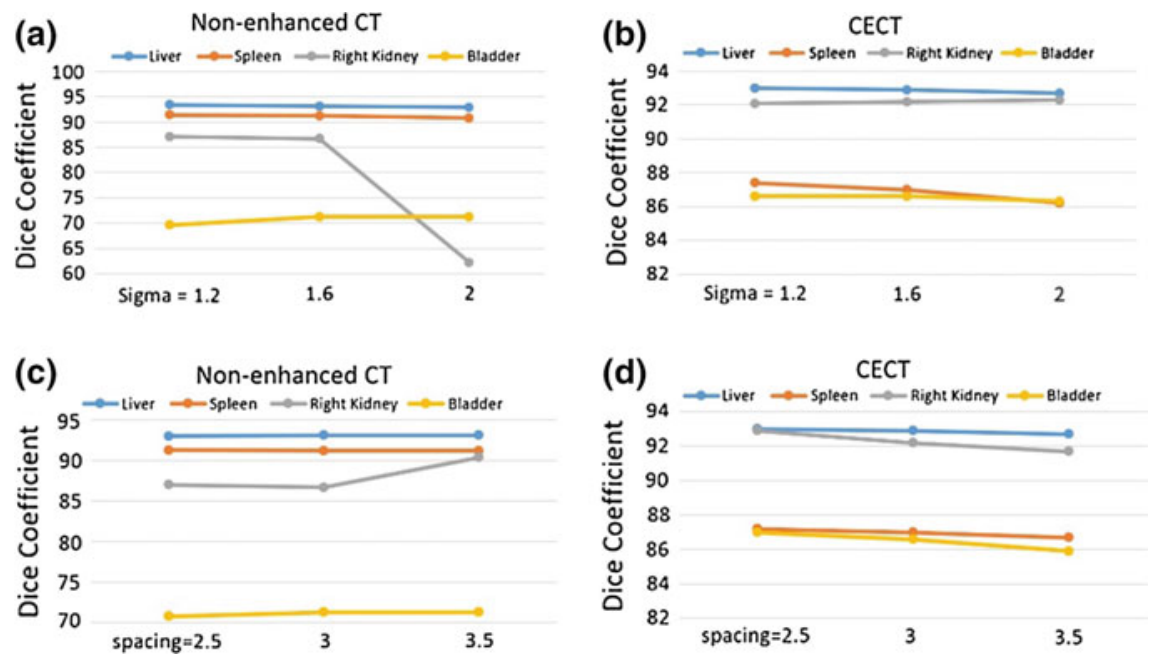

Fig. 10.9 a, b Plot of the segmentation accuracy measured with Dice coefficient against the size of the smoothing kernel (measured in voxels). c, $\mathbf{d}$ Plot of the segmentation accuracy against the image resolution used for the segmentation (measured in $\mathrm{mm}$ )

The average processing time for segmenting 10 selected organs was about $15 \mathrm{~min}$ for Implementation 1, $25 \mathrm{~min}$ for Implementation 2 and $55 \mathrm{~min}$ for Implementation 3, when running on a PC with Intel i7 $(1.9 \mathrm{GHz})$. A $7 \times 7 \times 7$ quadrature filter with a central frequency of $\pi / 2$ and a bandwidth of 6 octaves was used for the experiments.

\subsection{Discussion and Conclusion}

In the VISCERAL Anatomy Segmentation Benchmark [8], the proposed method outperformed conventional methods in terms of accuracy for brain stripping and liver, spleen and kidney segmentation tasks. Compared with other registration-based multi-atlas approaches that delivered superior results on some other structures [8], 
the proposed method has the advantage of being more computationally efficient. It is also interesting to note that some single-organ approaches fall behind the multiorgan approaches in terms of accuracy. This, to some extent, proves our hypothesis that it is easier to tell the membership of voxel in a multiorgan setup than looking at a single organ.

In our experience, image smoothing is an inevitable step for our method to be able to work on non-contrast-enhanced CT, as these images are very noisy. Using a large smoothing kernel may help the active contours to avoid stopping prematurely. However, when the organs' intensities are very similar, using a large smoothing kernel may destroy the vague edges between two organs and lead to total failure. This could partly explain the relatively random results plotted in Fig. 10.9a. When dealing with contrast-enhanced CT images with high signal-to-noise ratio (SNR), the smoothing kernel applied before the image segmentation in general has a negative effect on the segmentation accuracy.

The downsampling rate is another key parameter to consider when balancing the segmentation accuracy and the processing time. As suggested by Fig. 10.9d, higher downsampling rates often lead to worse segmentation results. This can also be seen when comparing the results from Implementation 1 and Implementation 2. However, for non-contrast-enhanced CT images, the downsampling itself will have some smoothing effect, and therefore, unpredicted sharp performance jumps/drops may be observed (e.g. Fig. 10.9c).

In this preliminary study, the training samples that were used to create the statistical shape models were arbitrarily chosen without carefully investigating the proper training sample selection strategy or variation enlarging methods mentioned in [7]. This is partly due to the fact that the current implementation of the training pipeline is not fully automated. Manual adjustment is involved in the registration and position linking steps. Although the segmentation results seem to be relatively accurate on the 18 testing datasets, we expect the results to degrade when applying the current shape models to a larger population. To implement a more sophisticated training sample selection strategy and build better statistical shape models using a larger training sample group have been planned.

So far, the proposed solution can only selectively segment 10 anatomical structures. While extending the current framework to segment more structures is relatively easy, it may not deliver satisfactory results for all of them. Two challenges are expected on some of the other smaller structures: great position variation and great shape variation. The proposed method relies on the initial position of the shape model being relatively close to the target organ. If the relative position of the parent structure is far from the child structure, the proposed method may, in extreme cases, miss the targeted organ entirely. We plan to solve this problem by introducing machine-learning-based organ detectors [14]. These detectors could also help us handle heterogeneous cases with varying scan ranges. On the other hand, the distance map-based statistical shape model used here may not be an ideal representation for all anatomical structures, in particular for those structures with high anatomical variation. Changing the statistical shape model to skeleton-based models may be more suitable for segmenting such structures, as suggested in $[15,21]$. 
In conclusion, the hierarchical shape model-guided multiorgan segmentation method is a promising approach to solve the ambiguity between two attaching organs. By introducing a model-based local phase term into the energy function and solving the minimization problem using our novel coherent propagation algorithm, we have demonstrated that the proposed multiorgan segmentation method can deliver accurate results using relatively short processing times.

\section{References}

1. Belaid A, Boukerroui D, Maingourd Y, Lerallut JF (2011) Phase-based level set segmentation of ultrasound images. IEEE Trans Inf Technol Biomed 15(1):138-147. doi:10.1109/TITB. 2010.2090889

2. Caselles V, Kimmel R, Sapiro G (1997) Geodesic active contours. Int J Comput Vis 22(1):6179. doi:10.1023/A:1007979827043

3. Chan TF, Vese LA (2001) Active contours without edges. IEEE Trans Image Process 10(2):266277. doi:10.1109/83.902291

4. Cootes T, Taylor C, Cooper D, Graham J (1995) Active shape models-their training and application. Comput Vis Image Underst 61(1):38-59. doi:10.1006/cviu.1995.1004

5. Cremers D, Rousson M, Deriche R (2007) A review of statistical approaches to level set segmentation: integrating color, texture, motion and shape. Int J Comput Vis 72(2):195-215. doi:10.1007/s11263-006-8711-1

6. Freeman WT, Adelson EH (1991) The design and use of steerable filters. IEEE Trans Pattern Anal Mach Intell 13(9):891-906. doi:10.1109/34.93808

7. Heimann T, Meinzer HP (2009) Statistical shape models for 3D medical image segmentation: a review. Med Image Anal 13(4):543-563. doi:10.1016/j.media.2009.05.004

8. Jiménez del Toro OJ, Müller H, Krenn M, Gruenberg K, Taha AA, Winterstein M, Eggel I, Foncubierta-Rodriguez A, Goksel O, Jakab A, Kontokotsios G, Langs G, Menze B, Fernandez TS, Schaer R, Walleyo A, Weber MA, Cid YD, Gass T, Heinrich M, Jia F, Kahl F, Kechichian R, Mai D, Spanier A, Vincent G, Wang C, Wyeth D, Hanbury A (2016) Cloud-based evaluation of anatomical structure segmentation and landmark detection algorithms: VISCERAL anatomy benchmarks. IEEE Trans Med Imaging. doi:10.1109/TMI.2016.2578680

9. Kainmueller D, Lange T, Lamecker H (2007) Shape constrained automatic segmentation of the liver based on a heuristic intensity model. In: Proceedings of MICCAI workshop on 3D segmentation in the clinic: a grand challenge, pp 109-116

10. Knutsson H (1994) Signal processing for computer vision. Springer, Berlin

11. Läthén G, Jonasson J, Borga M (2010) Blood vessel segmentation using multi-scale quadrature filtering. Pattern Recognit Lett 31(8):762-767. doi:10.1016/j.patrec.2009.09.020

12. Lefohn AE, Cates JE, Whitaker RT (2003) Interactive, GPU-based level sets for 3D segmentation. In: Ellis RE, Peters TM (eds) Proceedings of the 6th international conference medical image computing and computer-assisted intervention-MICCAI, Montréal, Canada, Nov 1518, 2003. Springer, Berlin, pp 564-572. doi:10.1007/978-3-540-39899-8_70

13. Leventon ME, Grimson WEL, Faugeras O (2000) Statistical shape influence in geodesic active contours. In: Proceedings of the IEEE conference on computer vision and pattern recognition, 2000, vol 1, pp 316-323. doi:10.1109/CVPR.2000.855835

14. Wang C, Lundström C (2016) CT scan range estimation using multiple body parts detection: let PACS learn the CT image content. Int J Comput Assist Radiol Surg 11(2):317-325. doi:10. 1007/s11548-015-1232-z

15. Wang C, Smedby Ö (2013) Fully automatic brain segmentation using model-guided level sets and skeleton-based models. MIDAS J 
16. Wang C, Smedby Ö (2014) Automatic multi-organ segmentation using fast model based level set method and hierarchical shape priors. In: Goksel O (ed) Proceedings of the VISCERAL challenge at ISBI, Beijing, China, no. 1194 in CEUR workshop proceedings, pp 25-31. http:// ceur-ws.org/Vol-1194/visceralISBI14-0.pdf

17. Wang C, Smedby Ö (2014) Automatic multi-organ segmentation in non-enhanced CT datasets using hierarchical shape priors. In: 2014 22nd international conference on pattern recognition (ICPR), pp 3327-3332. doi:10.1109/ICPR.2014.574

18. Wang C, Smedby Ö (2014) Model-based left ventricle segmentation in 3D ultrasound using phase image. MIDAS J

19. Wang C, Smedby Ö (2015) Multi-organ segmentation using shape model guided local phase analysis. Springer, Berlin, pp 149-156. doi:10.1007/978-3-319-24574-4_18

20. Wang C, Frimmel H, Smedby O (2011) Level-set based vessel segmentation accelerated with periodic monotonic speed function. In: Proceedings of the SPIE medical imaging conference, p 79621M. doi:10.1117/12.876704

21. Wang C, Moreno R, Smedby Ö (2012) Vessel segmentation using implicit model-guided level sets. In: Proceedings of the 3D cardiovascular imaging: a MICCAI segmentation challenge workshop

22. Wang C, Frimmel H, Smedby O (2014) Fast level-set based image segmentation using coherent propagation. Med Phys 41(7):073501. doi:10.1118/1.4881315

23. Wang C, Dahlström N, Fransson SG, Lundström C, Smedby Ö (2015) Real-time interactive 3D tumor segmentation using a fast level-set algorithm. J Med Imaging Health Inf 5(8):1998-2002. doi:10.1166/jmihi.2015.1685

24. Whitaker RT (1998) A level-set approach to 3D reconstruction from range data. Int J Comput Vis 29(3):203-231. doi:10.1023/A:1008036829907

25. Zheng Y, Barbu A, Georgescu B, Scheuering M, Comaniciu D (2008) Four-chamber heart modeling and automatic segmentation for 3-D cardiac CT volumes using marginal space learning and steerable features. IEEE Trans Med Imaging 27(11):1668-1681. doi:10.1109/TMI.2008. 2004421

Open Access This chapter is licensed under the terms of the Creative Commons Attribution- NonCommercial 2.5 International License (http://creativecommons.org/licenses/by-nc/2.5/), which permits any noncommercial use, sharing, adaptation, distribution and reproduction in any medium or format, as long as you give appropriate credit to the original author(s) and the source, provide a link to the Creative Commons license and indicate if changes were made.

The images or other third party material in this chapter are included in the chapter's Creative Commons license, unless indicated otherwise in a credit line to the material. If material is not included in the chapter's Creative Commons license and your intended use is not permitted by statutory regulation or exceeds the permitted use, you will need to obtain permission directly from the copyright holder.

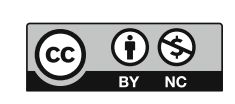

